Brief paper

\title{
Transmission scheduling for remote state estimation and control with an energy harvesting sensor
}

\author{
Alex S. Leong ${ }^{\mathrm{a}, *}$, Subhrakanti Dey ${ }^{\mathrm{b}}$, Daniel E. Quevedo ${ }^{\mathrm{a}}$ \\ a Department of Electrical Engineering (EIM-E), Paderborn University, Paderborn, Germany \\ ${ }^{\mathrm{b}}$ Institute for Telecommunications Research, University of South Australia, Adelaide, Australia
}

\section{A R T I C L E I N F O}

\section{Article history:}

Received 11 August 2016

Received in revised form 11 October 2017

Accepted 7 December 2017

\begin{abstract}
A B S T R A C T
This paper studies a remote state estimation problem where a sensor, equipped with energy harvesting capabilities, observes a dynamical process and transmits local state estimates over a packet dropping channel to a remote estimator. The objective is to decide, at every discrete time instant, whether the sensor should transmit or not, in order to minimize the expected estimation error covariance at the remote estimator over a finite horizon, subject to constraints on the sensor's battery energy governed by an energy harvesting process. We establish structural results on the optimal scheduling which show that, for a given battery energy level and a given harvested energy, the optimal policy is a threshold policy on the error covariance. Similarly, for a given error covariance and a given harvested energy, the optimal policy is a threshold policy on the current battery level. An extension to the problem of transmission scheduling and control with an energy harvesting sensor is also considered.
\end{abstract}

(c) 2018 Elsevier Ltd. All rights reserved.

\section{Introduction}

The harvesting of energy from the surrounding environment, such as solar, thermal, mechanical vibrations, or electromagnetic radiation, has attracted significant research interest, due to its potential for enabling self-sustaining and environmentally friendly devices. In wireless communications, transmission strategies for optimizing communication objectives such as maximizing throughput or minimizing transmission delay have been extensively studied, see e.g. Ho and Zhang (2012), Ozel, Tutuncuoglu, Yang, Ulukus, and Yener (2011) and Sharma, Mukherji, Joseph, and Gupta (2010). In the control literature, power/energy allocation strategies for optimizing state estimation (Li, Quevedo, Lau, Dey, \& Shi, 2017; Nourian, Leong, \& Dey, 2014) and control (Knorn \& Dey, 2017) objectives have also received recent attention.

In event triggered estimation, a sensor will transmit to a remote estimator only when certain events occur, e.g. if the estimation quality has deteriorated sufficiently, with different transmission strategies proposed (Li, Lemmon, \& Wang, 2010; Trimpe \& D’Andrea, 2014; Wu, Jia, Johansson, \& Shi, 2013; Xia, Gupta,

\footnotetext{
The material in this paper was partially presented at the 24th EUSIPCO 2016, 29 August-2 September, 2016, Budapest, Hungary. This paper was recommended for publication in revised form by Associate Editor Shreyas Sundaram under the direction of Editor Christos G. Cassandras.

* Corresponding author.

E-mail addresses: alex.leong@upb.de (A.S. Leong), Subhra.Dey@unisa.edu.au (S. Dey), dquevedo@ieee.org (D.E. Quevedo).
}

\& Antsaklis, 2013). A probabilistic triggering mechanism has also been recently studied in event triggered estimation with an energy harvesting sensor (Huang, Shi, \& Chen, 2017).

In this paper we will study a transmission scheduling problem for remote state estimation, that minimizes the expected estimation error covariance at the remote estimator. The scheduling is subject to the constraint that the sensor is equipped with energy harvesting capabilities, and transmission over a packet dropping channel can only occur if there is sufficient energy in the sensor battery. Note that one can regard the situation where there is insufficient battery energy for transmission as a sensor failure. Other related work on sensor failures include Chen, Yu, Zhang, and Liu (2013), Hounkpevi and Yaz (2007), Qu and Zhou (2013), Wang, Ho, and Liu (2003), to mention a few.

We will derive structural results on the optimal transmission policy. Namely, for a given battery energy level and a given harvested energy, we will show that the optimal policy is a threshold policy on the estimation error covariance. Similarly, for a given error covariance and a given harvested energy, the optimal policy is a threshold policy on the battery level. This is reminiscent of the threshold based policies often considered in event triggered estimation. We then extend our results to the problem of transmission scheduling and control with an energy harvesting sensor, where one can show that this problem is separable into an LQG-type control problem and a transmission scheduling problem, with the optimal transmission schedule having threshold-type behaviour.

Optimality of threshold-type policies has been shown in other contexts. For the case of noiseless measurements and no packet 


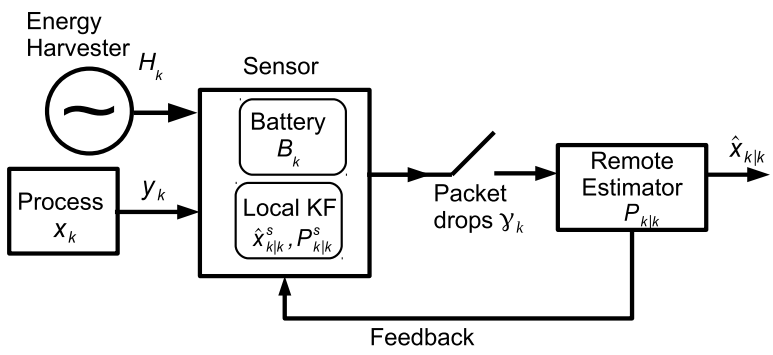

Fig. 1. Remote state estimation with an energy harvesting sensor.

drops, Lipsa and Martins (2011) showed a threshold behaviour in the difference between the current state and most recently transmitted state, with Nayyar, Başar, Teneketzis, and Veeravalli (2013) also considering energy harvesting. In event triggered control, optimality of threshold policies have been shown in Molin and Hirche (2010). For variance based triggering (where transmit decisions depend on the estimation error covariance) with no energy harvesting, it was shown in Leong, Dey, and Quevedo (2017) (see also Mo, Sinopoli, Shi, \& Garone, 2012; Ren, Cheng, Chen, Shi, \& Zhang, 2014) that threshold-type policies are optimal, in the sense that it minimizes a linear combination of the expected estimation error covariance and expected energy usage of the sensors.

The paper is organized as follows. Section 2 describes the system model. The optimal transmission scheduling problem is formulated in Section 3. Structural results for the optimal transmission schedule are derived in Section 4. The problem of transmission scheduling for control with an energy harvesting sensor is considered in Section 5. Numerical studies are presented in Section 6.

\section{System model}

A diagram of the system model is shown in Fig. 1. Consider a discrete time process

$x_{k+1}=A x_{k}+w_{k}$

where $x_{k} \in \mathbb{R}^{n_{x}}$ and $w_{k}$ is i.i.d. Gaussian with zero mean and covariance $Q \geq 0 .{ }^{1}$ There is a sensor taking measurements

$y_{k}=C x_{k}+v_{k}$,

where $y_{k} \in \mathbb{R}^{n_{y}}$ and $v_{k}$ is Gaussian with zero mean and covariance $R>0$. The noise processes $\left\{w_{k}\right\}$ and $\left\{v_{k}\right\}$ are assumed to be mutually independent.

The sensor has some computational capabilities and can run a local Kalman filter. The local state estimates and estimation error covariances

$$
\begin{aligned}
\hat{x}_{k \mid k-1}^{s} & \triangleq \mathbb{E}\left[x_{k} \mid y_{0}, \ldots, y_{k-1}\right], \hat{x}_{k \mid k}^{s} \triangleq \mathbb{E}\left[x_{k} \mid y_{0}, \ldots, y_{k}\right] \\
P_{k \mid k-1}^{s} & \triangleq \mathbb{E}\left[\left(x_{k}-\hat{x}_{k \mid k-1}^{s}\right)\left(x_{k}-\hat{x}_{k \mid k-1}^{s}\right)^{T} \mid y_{0}, \ldots, y_{k-1}\right] \\
P_{k \mid k}^{s} & \triangleq \mathbb{E}\left[\left(x_{k}-\hat{x}_{k \mid k}^{s}\right)\left(x_{k}-\hat{x}_{k \mid k}^{s}\right)^{T} \mid y_{0}, \ldots, y_{k}\right]
\end{aligned}
$$

can be computed at the sensor using the standard Kalman filtering equations. We assume that the pair $(A, C)$ is detectable and the pair $\left(A, Q^{1 / 2}\right)$ is stabilizable, with the local Kalman filter operating in steady state, ${ }^{2}$ i.e. $P_{k \mid k}^{s}=\bar{P}, \forall k$, where $\bar{P}$ is the steady state value of $P_{k \mid k}^{s}$, which exists by the detectability assumption.

\footnotetext{
1 For a symmetric matrix $X$, we say that $X>0$ if it is positive definite, and $X \geq 0$ if it is positive semi-definite. Given two symmetric matrices $X$ and $Y$, we say that $X \leq Y$ if $Y-X$ is positive semi-definite, and $X<Y$ if $Y-X$ is positive definite.

2 The local Kalman filter in general converges to steady state at an exponential rate.
}

Let $v_{k} \in\{0,1\}$ be decision variables such that $v_{k}=1$ if and only if $\hat{x}_{k \mid k}^{s}$ is to be transmitted ${ }^{3}$ by the sensor to the remote estimator at time $k$. Let $B_{k}$ denote the battery level of the sensor at time $k$, with $B_{\max }$ the maximum capacity of the sensor's battery. There is an energy usage of $E$ for each scheduled transmission. Transmission at time $k$ can only occur if there is sufficient energy in the battery, i.e. $v_{k}=1$ is possible only when $B_{k} \geq E$. The sensor is equipped with energy harvesting capabilities, with the energy harvested by the sensor between the discrete time instants $k-1$ and $k$ denoted by $H_{k}$. Similar to Ho and Zhang (2012), the evolution of the battery level is modelled as

$B_{k+1}=\min \left\{B_{k}-v_{k} E+H_{k+1}, B_{\max }\right\}$,

with $v_{k}=0$ if $B_{k}<E$. The harvested energy process $\left\{H_{k}\right\}$ can in general be temporally correlated, e.g. the amount of solar energy harvested may differ significantly depending on the time of day and weather conditions (Ho \& Zhang, 2012). In this paper we will assume that $\left\{H_{k}\right\}$ is Markovian. We denote the support of $\left\{H_{k}\right\}$ by $\mathbb{H}$, and that of $B_{k}$ by $\mathbb{B} \subseteq\left[0, B_{\max }\right]$.

At time instances when $v_{k}=1$, the sensor transmits its local state estimate $\hat{x}_{k \mid k}^{s}$ over a packet dropping channel, see Fig. 1. Let $\gamma_{k} \in\{0,1\}$ be random variables such that $\gamma_{k}=1$ if and only if the transmission at time $k$ is successfully received by the remote estimator. We will assume that $\left\{\gamma_{k}\right\}$ is i.i.d. Bernoulli with

$\mathbb{P}\left(\gamma_{k}=1\right)=\lambda \in(0,1)$.

At instances where $v_{k}=1$, it is assumed that the remote estimator knows whether the transmission was successful or not, i.e., the remote estimator knows the value $\gamma_{k}$, with dropped packets discarded. Define

$$
\mathcal{I}_{k} \triangleq\left\{v_{0}, \ldots, v_{k}, v_{0} \gamma_{0}, \ldots, v_{k} \gamma_{k}, v_{0} \gamma_{0} \hat{x}_{0 \mid 0}^{s}, \ldots, v_{k} \gamma_{k} \hat{x}_{k \mid k}^{s}\right\}
$$

as the information set available to the remote estimator at time $k$. Denote the state estimates and error covariances at the remote estimator by:

$\hat{x}_{k \mid k} \triangleq \mathbb{E}\left[x_{k} \mid \mathcal{I}_{k}\right], \quad P_{k \mid k} \triangleq \mathbb{E}\left[\left(x_{k}-\hat{x}_{k \mid k}\right)\left(x_{k}-\hat{x}_{k \mid k}\right)^{T} \mid \mathcal{I}_{k}\right]$.

Given that the decision variables $v_{k}$ depend on $P_{k-1 \mid k-1}, H_{k}$ and $B_{k}$, but not on the state $x_{k}$, the optimal remote estimator can be shown to have the following form, similar to Xu and Hespanha (2005):

$$
\begin{aligned}
& \hat{x}_{k \mid k}=\left\{\begin{array}{cl}
A \hat{x}_{k-1 \mid k-1}, & v_{k} \gamma_{k}=0 \\
\hat{x}_{k \mid k}^{s}, & v_{k} \gamma_{k}=1
\end{array}\right. \\
& P_{k \mid k}=\left\{\begin{array}{cl}
f\left(P_{k-1 \mid k-1}\right) & , v_{k} \gamma_{k}=0 \\
\bar{P} & , v_{k} \gamma_{k}=1,
\end{array}\right.
\end{aligned}
$$

where

$f(X) \triangleq A X A^{T}+Q$.

We assume that $\gamma_{k}$ is fed back to the sensor before the transmission decision at the next time instant $k+1$. Thus, the remote estimate $P_{k \mid k}$ can be reconstructed at the sensor with this acknowledgement mechanism. ${ }^{4}$

Define the countable set

$\mathcal{S}=\left\{\bar{P}, f(\bar{P}), f^{2}(\bar{P}), \ldots\right\}$,

where $f^{n}($.$) is the n$-fold composition of $f($.$) , with the convention$ that $f^{0}(X)=X$. Then it is clear from (5) that $\mathcal{S}$ consists of all

\footnotetext{
3 When there are packet drops, sending state estimates generally gives better estimation performance than sending measurements (Xu \& Hespanha, 2005).

4 The case of imperfect feedback acknowledgements can also be considered, using similar ideas as in Nourian et al. (2014).
} 
possible values of $P_{k \mid k}$ at the remote estimator. There is a total ordering on the elements of $\mathcal{S}$ given by (see e.g. Shi and Zhang (2012))

$\bar{P} \leq f(\bar{P}) \leq f^{2}(\bar{P}) \leq \cdots$.

\section{Optimal transmission scheduling}

In this section we will formulate an optimization problem for determining the transmission schedule, that minimizes the expected error covariance of the remote estimator over a finite horizon, subject to energy harvesting constraints at the sensor.

As mentioned in Section 2, we will consider transmission policies where $v_{k}$ depends on $P_{k-1 \mid k-1}, B_{k}$, and $H_{k}$. We will consider the following optimization problem of finite horizon $K$ :

$$
\begin{aligned}
& \min _{\left\{v_{1}, \ldots, v_{K}\right\}} \sum_{k=1}^{K} \mathbb{E}\left[\operatorname{tr} P_{k \mid k}\right] \\
& =\min _{\left\{\nu_{1}, \ldots, v_{K}\right\}} \sum_{k=1}^{K} \mathbb{E}\left[\mathbb{E}\left[\operatorname{tr} P_{k \mid k} \mid P_{k-1 \mid k-1}, B_{k}, H_{k}, v_{k}\right]\right]
\end{aligned}
$$

subject to the energy harvesting constraints:

$v_{k} E \leq B_{k}, B_{k+1}=\min \left\{B_{k}-v_{k} E+H_{k+1}, B_{\max }\right\}$,

$\forall k \in\{1, \ldots, K\}$. Note that if for some $\kappa \in\{1, \ldots, K\}$ we have $v_{\kappa}=1$, then

$$
\begin{aligned}
& \mathbb{E}\left[\operatorname{tr} P_{\kappa \mid \kappa} \mid P_{\kappa-1 \mid \kappa-1}, v_{\kappa}, H_{\kappa}, B_{\kappa}\right] \\
& =v_{\kappa}\left[\lambda \operatorname{tr}(\bar{P})+(1-\lambda) \operatorname{tr} f\left(P_{\kappa-1 \mid \kappa-1}\right)\right]+\left(1-v_{\kappa}\right) \operatorname{trf}\left(P_{\kappa-1 \mid \kappa-1}\right) \\
& =v_{\kappa} \lambda \operatorname{tr}(\bar{P})+\left(1-v_{\kappa} \lambda\right) \operatorname{tr} f\left(P_{\kappa-1 \mid \kappa-1}\right),
\end{aligned}
$$

whereas if $v_{\kappa}=0$, then

$\mathbb{E}\left[\mathbb{E}\left[\operatorname{tr} P_{\kappa \mid \kappa} \mid P_{\kappa-1 \mid \kappa-1}, B_{\kappa}, H_{\kappa}, v_{\kappa}\right]\right]=A P_{\kappa-1 \mid \kappa-1} A^{T}+Q$.

Let the functions $J_{k}(\cdot, \cdot, \cdot): \mathcal{S} \times \mathbb{H} \times \mathbb{B} \rightarrow \mathbb{R}$ be defined recursively as:

$$
\begin{aligned}
& J_{K+1}(P, H, B)=0 \\
& J_{k}(P, H, B)=\min _{\substack{v \in\{0,1\} \\
v E \leq B}}\{v \lambda \operatorname{tr}(\bar{P})+(1-v \lambda) \operatorname{tr} f(P) \\
& \quad+v \lambda \mathbb{E}\left[J_{k+1}(\bar{P}, \tilde{H}, b(B-v E+\tilde{H})) \mid H\right] \\
& \left.\quad+(1-v \lambda) \mathbb{E}\left[J_{k+1}(f(P), \tilde{H}, b(B-v E+\tilde{H})) \mid H\right]\right\}
\end{aligned}
$$

for $k=K, \ldots, 1$, where the conditional expectations are with respect to $\tilde{H}$ given $H$ (with $\tilde{H}$ representing the harvested energy at time $k+1$ given that $H_{k}=H$ ), and $b($.$) is defined as$

$b(x) \triangleq \min \left\{x, B_{\max }\right\}$.

Problem (8) can be solved using the dynamic programming algorithm, by computing $J_{k}\left(P_{k-1 \mid k-1}, H_{k}, B_{k}\right)$ for $k=K, K-1, \ldots, 1$, after discretization of $\mathbb{H}$ and $\mathbb{B}$ if needed (e.g. if $H_{k}$ and $B_{k}$ are continuous valued).

\section{Structural properties of optimal transmission schedule}

In this section, we will derive structural results on the optimal solutions to problem (8). Specifically, we show in Theorem 4.3 that for given $B_{k}$ and $H_{k}$, the optimal policy is a threshold policy with respect to the error covariance $P_{k-1 \mid k-1}$, i.e. it is optimal to transmit if and only if $P_{k-1 \mid k-1}$ exceeds a certain threshold $P_{k}^{*}$ (dependent on $k, B_{k}$ and $H_{k}$ ). Similarly, for fixed $P_{k-1 \mid k-1}$ and $H_{k}$, the optimal policy is a threshold policy with respect to the battery level $B_{k}$.
We will say that a function $F():. \mathcal{S} \rightarrow \mathbb{R}$ is increasing if

$X \leq Y \Rightarrow F(X) \leq F(Y)$.

Lemma 4.1. For any $n \in \mathbb{N}$, the function $F(P)=\operatorname{tr}\left(f^{n}(P)\right)$ is an increasing function of $P$.

Proof. We have

$F(P)=\operatorname{tr}\left(f^{n}(P)\right)=\operatorname{tr}\left(A^{n} P\left(A^{n}\right)^{T}+\sum_{m=0}^{n-1} A^{m} Q\left(A^{m}\right)^{T}\right)$.

As $P_{1} \leq P_{2} \Rightarrow A^{n} P_{1}\left(A^{n}\right)^{T} \leq A^{n} P_{2}\left(A^{n}\right)^{T} \Rightarrow \operatorname{tr}\left(A^{n} P_{1}\left(A^{n}\right)^{T}\right) \leq$ $\operatorname{tr}\left(A^{n} P_{2}\left(A^{n}\right)^{T}\right)$, the result follows.

Lemma 4.2. For $d \geq 0, b($.$) defined in (13) satisfies$

$0 \leq b(x)-b(x-d) \leq d$.

Proof. The inequality $b(x)-b(x-d) \geq 0$ is obvious. For the other inequality, note that if $x \leq B_{\max }$, then $b(x)-b(x-d)=x-(x-d)=$ $d$. If $x>B_{\max }$ and $x-d>B_{\max }$, then $b(x)-b(x-d)=B_{\max }-B_{\max }=$ 0 . If $x>B_{\max }$ (which implies $x-d>B_{\max }-d$ ) and $x-d \leq B_{\max }$, then $b(x)-b(x-d)=B_{\max }-(x-d)<B_{\max }-\left(B_{\max }-d\right)=d$.

Theorem 4.3. (i) For fixed $B_{k}$ and $H_{k}$, the optimal $v_{k}^{*}$ to problem (8) is of the form:

$v_{k}^{*}\left(P_{k-1 \mid k-1}, B_{k}, H_{k}\right)= \begin{cases}0, & P_{k-1 \mid k-1} \leq P_{k}^{*} \\ 1, & \text { otherwise }\end{cases}$

where the threshold $P_{k}^{*} \in \mathcal{S}$ depends on $k, B_{k}$ and $H_{k}$.

(ii) For fixed $P_{k-1 \mid k-1}$ and $H_{k}$, the optimal $v_{k}^{*}$ to problem (8) is of the form:

$v_{k}^{*}\left(P_{k-1 \mid k-1}, B_{k}, H_{k}\right)= \begin{cases}0, & B_{k} \leq B_{k}^{*} \\ 1, & \text { otherwise }\end{cases}$

where $B_{k}^{*} \in \mathbb{R}$ depends on $k, P_{k-1 \mid k-1}$ and $H_{k}$.

Proof. See Appendix.

Remark 4.4. Determining the actual values of $P_{k}^{*}$ and $B_{k}^{*}$, in general needs to be done numerically, e.g. by solving problem (8) via (12).

By making use of both parts (i) and (ii) of Theorem 4.3, we see that for a given $k$ and $H_{k}$, the region of possible values of $\left(P_{k-1 \mid k-1}, B_{k}\right)$ can be divided into a "transmit" and "don't transmit" region, separated by a staircase-like threshold, see Fig. 3.

Knowing that the optimal policies are of threshold-type simplifies real-time implementation. In addition, specialized algorithms can be derived which can provide computational savings when solving problem (8) numerically. For example, for a given $\left(k, H_{k}\right)$, suppose we want to compute $v_{k}^{*}\left(P_{k-1 \mid k-1}, B_{k}, H_{k}\right)$ for all possible $\left(P_{k-1 \mid k-1}, B_{k}\right)$. Let $\mathbb{B}^{\prime}$ be equal to $\mathbb{B}$ if $\mathbb{B}$ is a finite set, otherwise let $\mathbb{B}^{\prime}$ denote a discretized version of $\mathbb{B}$. Without structural information, one would need to compare the values $v_{k}=0$ and $v_{k}=1$ at each $\left(P_{k-1 \mid k-1}, B_{k}\right)$, which results in around $(k+1) \times\left|\mathbb{B}^{\prime}\right|$ comparisons, where $\left|\mathbb{B}^{\prime}\right|$ is the cardinality of $\mathbb{B}^{\prime}$. However, by using structural information, one way to determine the staircase-like threshold is as follows. For the smallest possible value of $P_{k-1 \mid k-1}$, which is $\bar{P}$, search (in decreasing order) starting from the largest value of $B_{k}$ (i.e. $\left.B_{\max }\right)$ to find the threshold $B_{k}^{*}\left(\bar{P}, H_{k}\right)$. Then for the next smallest value of $P_{k-1 \mid k-1}$, which is $f(\bar{P})$, the threshold $B_{k}^{*}\left(f(\bar{P}), H_{k}\right)$ satisfies $B_{k}^{*}\left(f(\bar{P}), H_{k}\right) \leq B_{k}^{*}\left(\bar{P}, H_{k}\right)$, so we can now search (in decreasing order) for $B_{k}^{*}\left(f(\bar{P}), H_{k}\right)$ starting from $B_{k}^{*}\left(\bar{P}, H_{k}\right)$ rather than from $B_{\max }$. Continuing this procedure until we cover all possible values of $P_{k-1 \mid k-1}$, it is not too difficult to show that the number of 


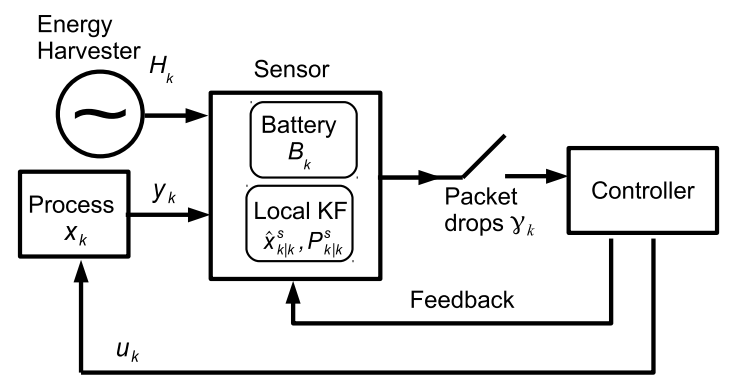

Fig. 2. Control with an energy harvesting sensor.

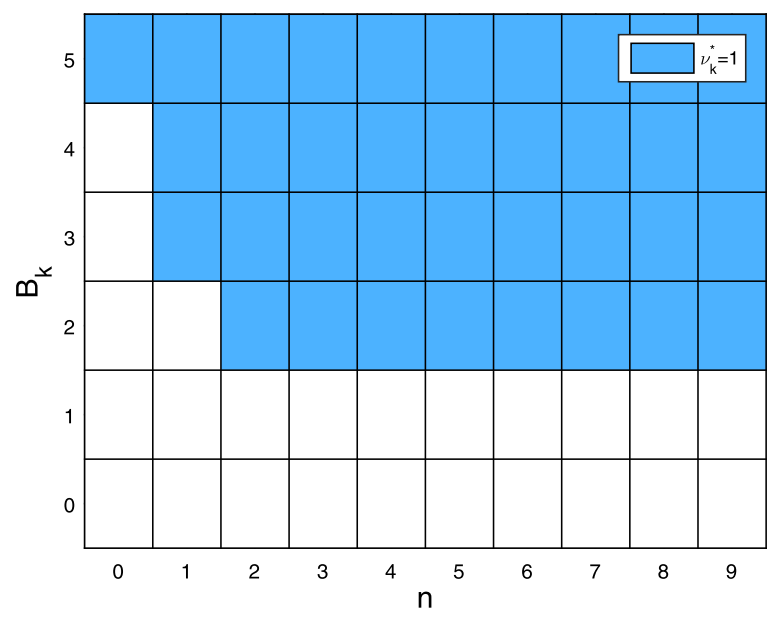

Fig. 3. $v_{k}^{*}$ for different values of $P_{k-1 \mid k-1}=f^{n}(\bar{P})$ and $B_{k}$, with $k=5$ and $H_{k}=1$.

comparisons required (for each $\left(k, H_{k}\right)$ ) to determine the staircaselike threshold is upper bounded by $2\left(k+1+\left|\mathbb{B}^{\prime}\right|\right)$. This could be significantly less than $(k+1) \times\left|\mathbb{B}^{\prime}\right|$, the number of comparisons needed if no structural information is assumed.

\section{Transmission scheduling for control with an energy harvest- ing sensor}

In this section we briefly describe an extension to the problem of transmission scheduling and control with an energy harvesting sensor. A diagram of the system model is shown in Fig. 2. The system model mostly follows that of Section 2, except that the discrete time process is now:

$x_{k+1}=A x_{k}+\mathfrak{B} u_{k}+w_{k}$,

where the control signal $u_{k} \in \mathbb{R}^{n_{u}}$.

We wish to solve the following problem:

$\min _{\substack{\left\{v_{1}, \ldots, v_{K}, u_{1}, \ldots, u_{K}\right\}}} \mathbb{E}\left[\sum_{k=1}^{K}\left(x_{k}^{T} W x_{k}+u_{k}^{T} U u_{k}\right)+x_{K+1}^{T} W x_{K+1}\right]$

subject to the energy harvesting constraints (9), where the matrices $W \geq 0$ and $U>0$. This problem minimizes the control cost (16) subject to the energy harvesting constraints (9), with the optimization being over both the controls $\left\{u_{k}\right\}$ and transmission decisions $\left\{v_{k}\right\}$. By following similar arguments as in Knorn and Dey (2017), we can show that for transmit decisions $v_{k}$ dependent only on $\left(P_{k-1 \mid k-1}, B_{k}, H_{k}\right)$, problem (16) is equivalent to:

$$
\min _{\left\{\nu_{1}, \ldots, v_{K}\right\}}\left[\min _{\left\{u_{1}, \ldots, u_{K}\right\}} \mathbb{E}\left[\sum_{k=1}^{K}\left(x_{k}^{T} W x_{k}+u_{k}^{T} U u_{k}\right)+x_{K+1}^{T} W x_{K+1}\right]\right],
$$

subject to (9). The inner optimization in (17) is an LQG-type problem with optimal cost $\operatorname{tr}\left(S_{1} P_{1}\right)+\sum_{k=1}^{K} \operatorname{tr}\left(S_{k+1} Q\right)+\sum_{k=1}^{K} \operatorname{tr}\left(\left(A^{T} S_{k+1}\right.\right.$ $\left.\left.A+W-S_{k}\right) \mathbb{E}\left[P_{k \mid k}\right]\right)$, where $S_{K+1}=W$,

$S_{k}=A^{T} S_{k+1} A+W-A^{T} S_{k+1} \mathfrak{B}\left(\mathfrak{B}^{T} S_{k+1} \mathfrak{B}+U\right)^{-1} \mathfrak{B}^{T} S_{k+1} A$, and $P_{1}$ is the covariance of $x_{1}$.

The optimal transmission schedule can then be found by solving the problem:

$$
\min _{\left\{v_{1}, \ldots, v_{K}\right\}}\left[\sum_{k=1}^{K} \operatorname{tr}\left(\left(A^{T} S_{k+1} A+W-S_{k}\right) \mathbb{E}\left[P_{k \mid k}\right]\right)\right]
$$

subject to (9). We have:

Theorem 5.1. (i) For fixed $B_{k}$ and $H_{k}$, the optimal $v_{k}^{*}$ to problem (18) is of the form:

$$
v_{k}^{*}\left(P_{k-1 \mid k-1}, B_{k}, H_{k}\right)= \begin{cases}0, & P_{k-1 \mid k-1} \leq \tilde{P}_{k}^{*} \\ 1, & \text { otherwise }\end{cases}
$$

where $\tilde{P}_{k}^{*} \in \mathcal{S}$ depends on $k, B_{k}$ and $H_{k}$.

(ii) For fixed $P_{k-1 \mid k-1}$ and $H_{k}$, the optimal $v_{k}^{*}$ to problem (18) is of the form:

$$
v_{k}^{*}\left(P_{k-1 \mid k-1}, B_{k}, H_{k}\right)= \begin{cases}0, & B_{k} \leq \tilde{B}_{k}^{*} \\ 1, & \text { otherwise }\end{cases}
$$

where $\tilde{B}_{k}^{*} \in \mathbb{R}$ depends on $k, P_{k-1 \mid k-1}$ and $H_{k}$.

Proof. Let us denote $G_{k} \triangleq A^{T} S_{k+1} A+W-S_{k}$. From the recursion for $S_{k}$ we have $G_{k}=A^{T} S_{k+1} \mathfrak{B}\left(\mathfrak{B}^{T} S_{k+1} \mathfrak{B}+U\right)^{-1} \mathfrak{B} S_{k+1} A$, which shows that $G_{k} \geq 0, \forall k$, since $S_{k+1} \geq 0$ and $U>0$. As $G_{k} \geq 0$, we can use Lemma 8.4.12 of Bernstein (2009) to extend Lemma 4.1 to show that $F(P)=\operatorname{tr}\left(G_{k} f^{n}(P)\right)$ is increasing in $P$ for all $k$ and all $n$. We may then verify that similar arguments used in the proof of Theorem 4.3 also apply to problem (18).

\section{Numerical studies}

We consider an example with parameters

$A=\left[\begin{array}{ll}1.2 & 0.2 \\ 0.2 & 0.7\end{array}\right], C=\left[\begin{array}{ll}1 & 1\end{array}\right], Q=I, R=1$,

in which case $\bar{P}$ is easily computed as

$\bar{P}=\left[\begin{array}{rr}1.3634 & -0.8347 \\ -0.8347 & 1.0809\end{array}\right]$.

The packet reception probability is chosen to be $\lambda=0.7$. The transmission energy $E=2$. The harvested energy process $\left\{H_{k}\right\}$ is chosen to be a Markov chain with state space $\{0,1,2\}$ and transition probability matrix

$$
\left[\begin{array}{lll}
p_{00} & p_{01} & p_{02} \\
p_{10} & p_{11} & p_{12} \\
p_{20} & p_{21} & p_{22}
\end{array}\right]=\left[\begin{array}{lll}
0.2 & 0.3 & 0.5 \\
0.3 & 0.4 & 0.3 \\
0.1 & 0.2 & 0.7
\end{array}\right]
$$

with the initial distribution of $H_{1}$ having the stationary distribution. The maximum battery capacity $B_{\max }=6$. We use the finite horizon $K=10$.

Fig. 3 plots $v_{k}^{*}$ for different values of $P_{k-1 \mid k-1}=f^{n}(\bar{P})$ and $B_{k}$, for fixed $k=5$ and $H_{k}=1$. We observe threshold-like behaviour in agreement with Theorem 4.3. Next, we consider the case where the maximum battery capacity $B_{\max }$ is varied. Fig. 4 plots the trace of the expected error covariance $\operatorname{tr} \mathbb{E}\left[P_{k \mid k}\right]$ vs. $B_{\max }$, with $\operatorname{tr} \mathbb{E}\left[P_{k \mid k}\right]$ obtained by averaging over 100000 Monte Carlo runs, with each run having the initial values $P_{0 \mid 0}=\bar{P}$ and $B_{1}=E$. We compare the performance with a simple suboptimal policy which always 


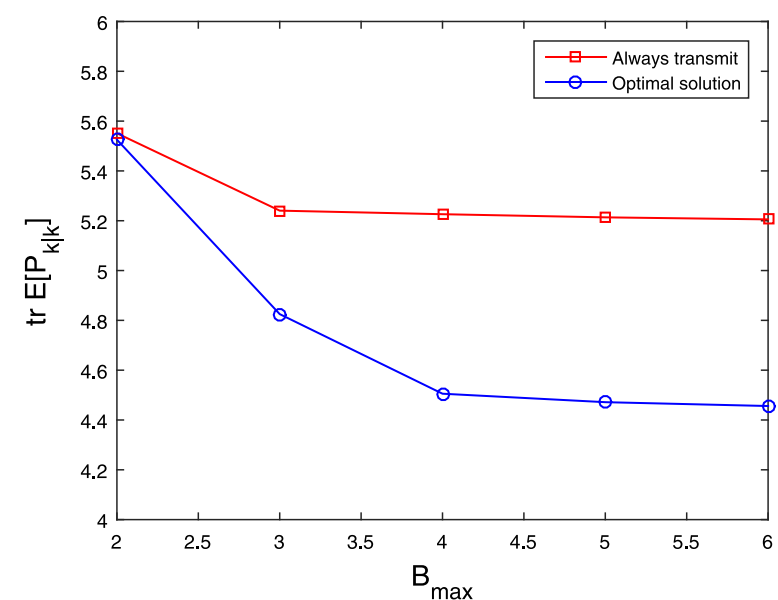

Fig. 4. Expected error covariance vs. $B_{\max }$.

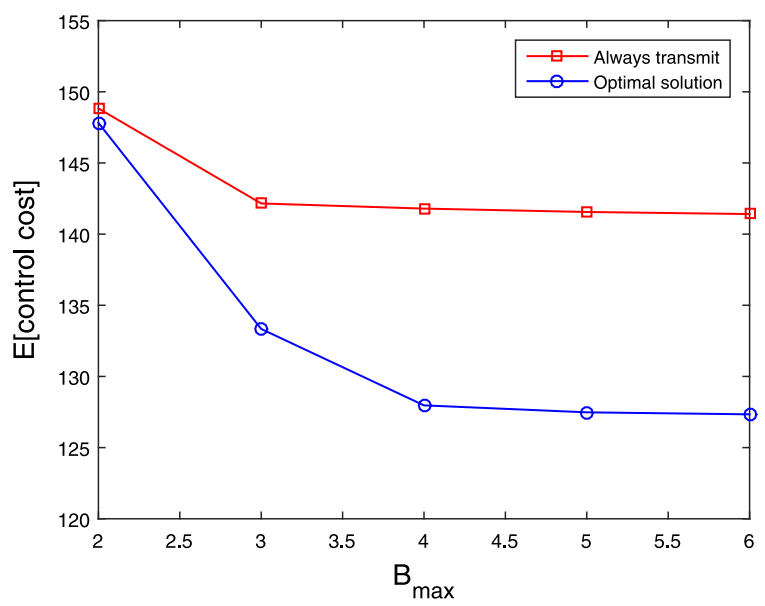

Fig. 5. Expected control cost vs. $B_{\max }$.

transmits provided it has enough energy. We see that the optimal policy significantly outperforms this suboptimal policy.

Finally, we consider the control problem of Section 5. We use the same parameters as before, together with $\mathfrak{B}=\left[\begin{array}{ll}1 & 2\end{array}\right]^{T}, W=I$, $U=1$. Fig. 5 plots the expected control cost $\mathbb{E}\left[\sum_{k=1}^{K}\left(x_{k}^{T} W x_{k}+\right.\right.$ $\left.\left.u_{k}^{T} U u_{k}\right)+x_{K+1}^{T} W x_{K+1}\right]$ vs. $B_{\max }$. We compare the performance with the policy which always transmits provided it has enough energy, which we again see is significantly outperformed by the optimal policy.

\section{Conclusion}

This paper has studied transmission scheduling problems for remote state estimation and control, where the sensor is equipped with energy harvesting capabilities, and transmission is over a stochastic packet dropping channel. We have derived structural results on the optimal transmission schedules, with thresholding behaviour in both the estimation error covariance and battery level.

\section{Appendix. Proof of Theorem 4.3}

We will prove part (i). The proof of part (ii) is omitted, since it can be proved using similar techniques as in the proof of Theorem 6.1 of Nourian et al. (2014).
For $B \geq E, J_{k}(P, H, B)$ in (12) can be expressed as

$$
\begin{aligned}
& J_{k}(P, H, B)=\min \left\{\operatorname{tr} f(P)+\mathbb{E}\left[J_{k+1}(f(P), \tilde{H}, b(B+\tilde{H})) \mid H\right],\right. \\
& \lambda \operatorname{tr} \bar{P}+(1-\lambda) \operatorname{tr} f(P)+\lambda \mathbb{E}\left[J_{k+1}(\bar{P}, \tilde{H}, b(B-E+\tilde{H})) \mid H\right] \\
& \left.\quad+(1-\lambda) \mathbb{E}\left[J_{k+1}(f(P), \tilde{H}, b(B-E+\tilde{H})) \mid H\right]\right\},
\end{aligned}
$$

where the two terms in the minimization correspond to the values $v_{k}=0$ or $v_{k}=1$. Since $v_{k}$ only takes on the two values 0 and 1 , Theorem 4.3 will be proved if we can show that for fixed $B \geq E$ and $H$, the functions

$$
\begin{aligned}
\phi_{k}(P) \triangleq \operatorname{tr} f(P)+\mathbb{E}\left[J_{k+1}(f(P), \tilde{H}, b(B+\tilde{H})) \mid H\right]-\lambda \operatorname{tr} \bar{P} \\
-(1-\lambda) \operatorname{tr} f(P)-\lambda \mathbb{E}\left[J_{k+1}(\bar{P}, \tilde{H}, b(B-E+\tilde{H})) \mid H\right] \\
-(1-\lambda) \mathbb{E}\left[J_{k+1}(f(P), \tilde{H}, b(B-E+\tilde{H})) \mid H\right], \\
=\lambda\left(\operatorname{tr} f(P)-\operatorname{tr} \bar{P}-\mathbb{E}\left[J_{k+1}(\bar{P}, \tilde{H}, b(B-E+\tilde{H})) \mid H\right]\right) \\
\quad+\mathbb{E}\left[J_{k+1}(f(P), \tilde{H}, b(B+\tilde{H})) \mid H\right] \\
\quad-(1-\lambda) \mathbb{E}\left[J_{k+1}(f(P), \tilde{H}, b(B-E+\tilde{H})) \mid H\right]
\end{aligned}
$$

for $k=1, \ldots, K$ are increasing functions of $P$. As $\operatorname{tr} f(P)$ is increasing with $P$ by Lemma 4.1, this will be the case if we can show that $\mathbb{E}\left[J_{k}(f(P), \tilde{H}, b(B+\tilde{H})) \mid H\right]-(1-\lambda) \mathbb{E}\left[J_{k}(f(P), \tilde{H}, b(B-E+\right.$ $\tilde{H})) \mid H]$ is an increasing function of $P$ for all $k$. To prove this using an induction argument, we will in fact prove a slightly stronger statement, namely that

$J_{k}\left(f^{n}(P), H, B\right)-(1-\lambda) J_{k}\left(f^{n}(P), H, B^{\prime}\right)$

is an increasing function of $P$ for all $k \in\{1, \ldots, K+1\}, n \in \mathbb{N}$, $H \geq 0, B \geq 0, B^{\prime} \geq 0$ with $0 \leq B-B^{\prime} \leq E$, noting that $0 \leq b(B+\tilde{\tilde{H}})-b(B-E+\tilde{H}) \leq E$ by Lemma 4.2 . In order to show that (A.1) is an increasing function of $P$, it turns out that we also need to show that

$J_{k}\left(f^{n}(P), H, B^{\prime}\right)-J_{k}\left(f^{n}(P), H, B\right)$

is an increasing function of $P$ for all $k \in\{1, \ldots, K+1\}, n \in \mathbb{N}$, $H \geq 0, B \geq 0, B^{\prime} \geq 0$ with $0 \leq B-B^{\prime} \leq E$.

As stated before, the proof is by induction. That (A.1) and (A.2) are increasing functions of $P$ in the case of $k=K+1$ is clear. For $P \geq P^{\prime}$ and $0 \leq B-B^{\prime} \leq E$, assume that

$$
\begin{aligned}
& J_{l}\left(f^{n}(P), H, B\right)-(1-\lambda) J_{l}\left(f^{n}(P), H, B^{\prime}\right) \\
& -J_{l}\left(f^{n}\left(P^{\prime}\right), H, B\right)+(1-\lambda) J_{l}\left(f^{n}\left(P^{\prime}\right), H, B^{\prime}\right) \geq 0
\end{aligned}
$$

and

$J_{l}\left(f^{n}(P), H, B^{\prime}\right)-J_{l}\left(f^{n}(P), H, B\right)$

$-J_{l}\left(f^{n}\left(P^{\prime}\right), H, B^{\prime}\right)+J_{l}\left(f^{n}\left(P^{\prime}\right), H, B\right) \geq 0$

hold for $l=K+1, K, \ldots, k+1$.

Let us first show that (A.3) holds for $l=k$. We have

$J_{k}\left(f^{n}(P), H, B\right)-(1-\lambda) J_{k}\left(f^{n}(P), H, B^{\prime}\right)$

$-J_{k}\left(f^{n}\left(P^{\prime}\right), H, B\right)+(1-\lambda) J_{k}\left(f^{n}\left(P^{\prime}\right), H, B^{\prime}\right)$

$=\min _{\nu, \nu E \leq B}\left\{v \lambda \operatorname{tr} \bar{P}+(1-v \lambda) \operatorname{tr} f^{n+1}(P)\right.$

$$
\begin{aligned}
& +v \lambda \mathbb{E}\left[J_{k+1}(\bar{P}, \tilde{H}, b(B-v E+\tilde{H})) \mid H\right] \\
& \left.+(1-v \lambda) \mathbb{E}\left[J_{k+1}\left(f^{n+1}(P), \tilde{H}, b(B-v E+\tilde{H})\right) \mid H\right]\right\}
\end{aligned}
$$

$-(1-\lambda) \min _{\nu, \nu E \leq B^{\prime}}\left\{\nu \lambda \operatorname{tr} \bar{P}+(1-\nu \lambda) \operatorname{tr} f^{n+1}(P)\right.$ 


$$
\begin{aligned}
& +v \lambda \mathbb{E}\left[J_{k+1}\left(\bar{P}, \tilde{H}, b\left(B^{\prime}-v E+\tilde{H}\right)\right) \mid H\right] \\
& \left.+(1-v \lambda) \mathbb{E}\left[J_{k+1}\left(f^{n+1}(P), \tilde{H}, b\left(B^{\prime}-v E+\tilde{H}\right)\right) \mid H\right]\right\} \\
& -\min _{\nu, v E \leq B}\left\{v \lambda \operatorname{tr} \bar{P}+(1-v \lambda) \operatorname{tr} f^{n+1}\left(P^{\prime}\right)\right. \\
& +v \lambda \mathbb{E}\left[J_{k+1}(\bar{P}, \tilde{H}, b(B-v E+\tilde{H})) \mid H\right] \\
& \left.+(1-v \lambda) \mathbb{E}\left[J_{k+1}\left(f^{n+1}\left(P^{\prime}\right), \tilde{H}, b(B-v E+\tilde{H})\right) \mid H\right]\right\} \\
& +(1-\lambda) \min _{v, \nu E \leq B^{\prime}}\left\{v \lambda \operatorname{tr} \bar{P}+(1-v \lambda) \operatorname{tr} f^{n+1}\left(P^{\prime}\right)\right. \\
& +v \lambda \mathbb{E}\left[J_{k+1}\left(\bar{P}, \tilde{H}, b\left(B^{\prime}-v E+\tilde{H}\right)\right) \mid H\right] \\
& \left.+(1-v \lambda) \mathbb{E}\left[J_{k+1}\left(f^{n+1}\left(P^{\prime}\right), \tilde{H}, b\left(B^{\prime}-v E+\tilde{H}\right)\right) \mid H\right]\right\} .
\end{aligned}
$$

If $B \geq E$ and $B^{\prime} \geq E$, then

$$
\begin{aligned}
& J_{k}\left(f^{n}(P), H, B\right)-(1-\lambda) J_{k}\left(f^{n}(P), H, B^{\prime}\right) \\
& -J_{k}\left(f^{n}\left(P^{\prime}\right), H, B\right)+(1-\lambda) J_{k}\left(f^{n}\left(P^{\prime}\right), H, B^{\prime}\right) \\
& \geq \min _{v}(1-v \lambda)\left\{\lambda\left[\operatorname{tr} f^{n+1}(P)-\operatorname{tr} f^{n+1}\left(P^{\prime}\right)\right]\right. \\
& \quad+\mathbb{E}\left[J_{k+1}\left(f^{n+1}(P), \tilde{H}, b(B-v E+\tilde{H})\right) \mid H\right] \\
& \quad-(1-\lambda) \mathbb{E}\left[J_{k+1}\left(f^{n+1}(P), \tilde{H}, b\left(B^{\prime}-v E+\tilde{H}\right)\right) \mid H\right] \\
& \quad-\mathbb{E}\left[J_{k+1}\left(f^{n+1}\left(P^{\prime}\right), \tilde{H}, b(B-v E+\tilde{H})\right) \mid H\right] \\
& \left.\quad+(1-\lambda) \mathbb{E}\left[J_{k+1}\left(f^{n+1}\left(P^{\prime}\right), \tilde{H}, b\left(B^{\prime}-v E+\tilde{H}\right)\right) \mid H\right]\right\} \geq 0,
\end{aligned}
$$

where the last inequality holds (for both cases $v^{*}=0$ and $v^{*}=1$ ) by Lemma 4.1 and the induction hypothesis (A.3), since $0 \leq b(B-$ $v E+\tilde{H})-b\left(B^{\prime}-v E+\tilde{H}\right) \leq E$ when $0 \leq B-B^{\prime} \leq E$. If $B<E$ and $B^{\prime}<E$, then

$$
\begin{aligned}
& J_{k}\left(f^{n}(P), H, B\right)-(1-\lambda) J_{k}\left(f^{n}(P), H, B^{\prime}\right) \\
& -J_{k}\left(f^{n}\left(P^{\prime}\right), H, B\right)+(1-\lambda) J_{k}\left(f^{n}\left(P^{\prime}\right), H, B^{\prime}\right) \\
& =\left\{\lambda\left[\operatorname{tr} f^{n+1}(P)-\operatorname{tr}^{n+1}\left(P^{\prime}\right)\right]\right. \\
& \quad+\mathbb{E}\left[J_{k+1}\left(f^{n+1}(P), \tilde{H}, b(B+\tilde{H})\right) \mid H\right] \\
& \quad-(1-\lambda) \mathbb{E}\left[J_{k+1}\left(f^{n+1}(P), \tilde{H}, b\left(B^{\prime}+\tilde{H}\right)\right) \mid H\right] \\
& \quad-\mathbb{E}\left[J_{k+1}\left(f^{n+1}\left(P^{\prime}\right), \tilde{H}, b(B+\tilde{H})\right) \mid H\right] \\
& \left.\quad+(1-\lambda) \mathbb{E}\left[J_{k+1}\left(f^{n+1}\left(P^{\prime}\right), \tilde{H}, b\left(B^{\prime}+\tilde{H}\right)\right) \mid H\right]\right\} \geq 0,
\end{aligned}
$$

by Lemma 4.1 and the induction hypothesis (A.3). If $B \geq E$ and $B^{\prime}<E$, then

$$
\begin{aligned}
& J_{k}\left(f^{n}(P), H, B\right)-(1-\lambda) J_{k}\left(f^{n}(P), H, B^{\prime}\right) \\
& -J_{k}\left(f^{n}\left(P^{\prime}\right), H, B\right)+(1-\lambda) J_{k}\left(f^{n}\left(P^{\prime}\right), H, B^{\prime}\right) \\
& \geq \min _{v}\left\{\lambda(1-v)\left[\operatorname{tr} f^{n+1}(P)-\operatorname{tr} f^{n+1}\left(P^{\prime}\right)\right]\right. \\
& \quad+(1-v \lambda) \mathbb{E}\left[J_{k+1}\left(f^{n+1}(P), \tilde{H}, b(B-v E+\tilde{H})\right) \mid H\right] \\
& \quad-(1-\lambda) \mathbb{E}\left[J_{k+1}\left(f^{n+1}(P), \tilde{H}, b\left(B^{\prime}+\tilde{H}\right)\right) \mid H\right]
\end{aligned}
$$

$$
\begin{aligned}
& -(1-v \lambda) \mathbb{E}\left[J_{k+1}\left(f^{n+1}\left(P^{\prime}\right), \tilde{H}, b(B-v E+\tilde{H})\right) \mid H\right] \\
& \left.+(1-\lambda) \mathbb{E}\left[J_{k+1}\left(f^{n+1}\left(P^{\prime}\right), \tilde{H}, b\left(B^{\prime}+\tilde{H}\right)\right) \mid H\right]\right\} .
\end{aligned}
$$

In the minimization above, if the optimal $v^{*}=0$, then $J_{k}\left(f^{n}(P)\right.$, $H, B)-(1-\lambda) J_{k}\left(f^{n}(P), H, B^{\prime}\right)-J_{k}\left(f^{n}\left(P^{\prime}\right), H, B\right)+(1-\lambda) J_{k}\left(f^{n}\left(P^{\prime}\right)\right.$, $\left.H, B^{\prime}\right) \geq 0$ by a similar argument as before. If instead $v^{*}=1$, then

$$
\begin{aligned}
& J_{k}\left(f^{n}(P), H, B\right)-(1-\lambda) J_{k}\left(f^{n}(P), H, B^{\prime}\right) \\
& -J_{k}\left(f^{n}\left(P^{\prime}\right), H, B\right)+(1-\lambda) J_{k}\left(f^{n}\left(P^{\prime}\right), H, B^{\prime}\right) \\
& \geq(1-\lambda) \mathbb{E}\left[J_{k+1}\left(f^{n+1}(P), \tilde{H}, b(B-E+\tilde{H})\right) \mid H\right] \\
& \quad-(1-\lambda) \mathbb{E}\left[J_{k+1}\left(f^{n+1}(P), \tilde{H}, b\left(B^{\prime}+\tilde{H}\right)\right) \mid H\right] \\
& \quad-(1-\lambda) \mathbb{E}\left[J_{k+1}\left(f^{n+1}\left(P^{\prime}\right), \tilde{H}, b(B-E+\tilde{H})\right) \mid H\right] \\
& \quad+(1-\lambda) \mathbb{E}\left[J_{k+1}\left(f^{n+1}\left(P^{\prime}\right), \tilde{H}, b\left(B^{\prime}+\tilde{H}\right)\right) \mid H\right] \geq 0
\end{aligned}
$$

where the last inequality now holds by induction hypothesis (A.4), since $0 \leq b\left(B^{\prime}+\tilde{H}\right)-b(B-E+\tilde{H}) \leq E$ when $0 \leq B-B^{\prime} \leq E$. This proves that (A.3) holds for $l=k$.

It remains to show that (A.4) holds for $l=k$, i.e. that

$J_{k}\left(f^{n}(P), H, B^{\prime}\right)-J_{k}\left(f^{n}(P), H, B\right)$

$$
-J_{k}\left(f^{n}\left(P^{\prime}\right), H, B^{\prime}\right)+J_{k}\left(f^{n}\left(P^{\prime}\right), H, B\right) \geq 0 \text {. }
$$

This can be done by using similar arguments as showing that (A.3) holds for $l=k$, and making use of both induction hypotheses (A.4) and (A.3). The details are omitted for brevity.

\section{References}

Bernstein, D. S. (2009). Matrix mathematics (2nd ed.). New Jersey: Princeton University Press.

Chen, B., Yu, L., Zhang, W.-A., \& Liu, A. (2013). Robust information fusion estimator for multiple delay-tolerant sensors with different failure rates. IEEE Transactions on Circuits and Systems-I: Regular Papers, 60(2), 401-414.

Ho, C. K., \& Zhang, R. (2012). Optimal energy allocation for wireless communications with energy harvesting constraints. IEEE Transactions on Signal Processing, 60(9), 4808-4818.

Hounkpevi, F. O., \& Yaz, E. E. (2007). Robust minimum variance linear state estimators for multiple sensors with different failure rates. Automatica, 43, 1274-1280.

Huang, J., Shi, D., \& Chen, T. (2017). Event-triggered state estimation with an energy harvesting sensor. IEEE Transactions on Automatic Control, 62(9), 4768-4775.

Knorn, S., \& Dey, S. (2017). Optimal energy allocation for linear control with packet loss under energy harvesting constraints. Automatica, 77, 259-267.

Leong, A. S., Dey, S., \& Quevedo, D. E. (2017). Sensor scheduling in variance based event triggered estimation with packet drops. IEEE Transactions on Automatic Control, 62(4), 1880-1895.

Li, L., Lemmon, M., \& Wang, X. (2010). Event-triggered state estimation in vector linear processes. In Proc. American contr. conf. (pp. 2138-2143). Baltimore, MD.

Li, Y., Quevedo, D. E., Lau, V., Dey, S., \& Shi, L. (2017). Power control of an energy harvesting sensor for remote state estimation. IEEE Transactions on Automatic Control, 62(1), 277-290.

Lipsa, G. M., \& Martins, N. C. (2011). Remote state estimation with communication costs for first-order LTI systems. IEEE Transactions on Automatic Control, 56(9), 2013-2025.

Mo, Y., Sinopoli, B., Shi, L., \& Garone, E. (2012). Infinite-horizon sensor scheduling for estimation over lossy networks. In Proc. IEEE conf. decision and control (pp. 3317-3322). Maui, HI.

Molin, A., \& Hirche, S. (2010). Structural characterization of optimal event-based controllers for linear stochastic systems. In Proc. IEEE conf. decision and control (pp. 3227-3233). Atlanta, GA.

Nayyar, A., Bașar, T., Teneketzis, D., \& Veeravalli, V. V. (2013). Optimal strategies for communication and remote estimation with an energy harvesting sensor. IEEE Transactions on Automatic Control, 58(9), 2246-2260.

Nourian, M., Leong, A. S., \& Dey, S. (2014). Optimal energy allocation for Kalman filtering over packet dropping links with imperfect acknowledgments and 
energy harvesting constraints. IEEE Transactions on Automatic Control, 59(8), 2128-2143.

Ozel, O., Tutuncuoglu, K., Yang, J., Ulukus, S., \& Yener, A. (2011). Transmission with energy harvesting nodes in fading wireless channels: optimal policies. IEEE Journal on Selected Areas in Communications, 29(8), 1732-1743.

Qu, X., \& Zhou, J. (2013). The optimal robust finite-horizon Kalman filtering for multiple sensors with different stochastic failure rates. Applied Mathematics Letters, 26, 80-86.

Ren, Z., Cheng, P., Chen, J., Shi, L., \& Zhang, H. (2014). Dynamic sensor transmission power scheduling for remote state estimation. Automatica, 50, 1235-1242.

Sharma, V., Mukherji, U., Joseph, V., \& Gupta, S. (2010). Optimal energy management policies for energy harvesting sensor nodes. IEEE Transactions on Wireless Communications, 9(4), 1326-1336.

Shi, L., \& Zhang, H. (2012). Scheduling two Gauss-Markov systems: an optimal solution for remote state estimation under bandwidth constraint. IEEE Transactions on Signal Processing, 60(4), 2038-2042.

Trimpe, S., \& D'Andrea, R. (2014). Event-based state estimation with variance-based triggering. IEEE Transactions on Automatic Control, 59(12), 3266-3281.

Wang, Z., Ho, D. W. C., \& Liu, X. (2003). Variance-constrained filtering for uncertain stochastic systems with missing measurements. IEEE Transactions on Automatic Control, 48(7), 1254-1258.

Wu, J., Jia, Q.-S., Johansson, K. H., \& Shi, L. (2013). Event-based sensor data scheduling: trade-off between communication rate and estimation quality. IEEE Transactions on Automatic Control, 58(4), 1041-1046.

Xia, M., Gupta, V., \& Antsaklis, P. J. (2013). Networked state estimation over a shared communication medium. In Proc. American contr. conf. (pp. 4134-4319). Washington, DC.

Xu, Y., \& Hespanha, J. P. (2005). Estimation under uncontrolled and controlled communications in networked control systems. In Proc. IEEE conf. decision and control (pp. 842-847). Seville, Spain.

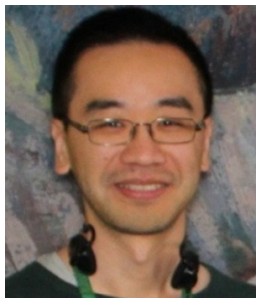

Alex S. Leong was born in Macau in 1980. He received the B.S. degree in mathematics and B.E. degree in electrical engineering in 2003, and the Ph.D. degree in electrical engineering in 2008, all from the University of Melbourne, Australia.

He is currently a Research Associate at Paderborn University, Germany. He was with the Department of Electrical and Electronic Engineering at the University of Melbourne from 2008 to 2015. His research interests include networked control systems, signal processing for sensor networks, and statistical signal processing.

Dr. Leong was the recipient of the L. R. East Medal from Engineers Australia in 2003, an Australian Postdoctoral Fellowship from the Australian Research Council in 2009, and a Discovery Early Career Researcher Award from the Australian Research Council in 2012.

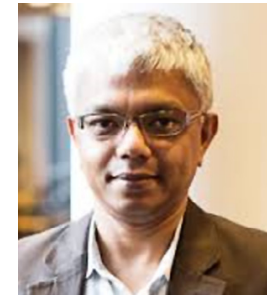

Subhrakanti Dey received the Bachelor in Technology and Master in Technology degrees from the Department of Electronics and Electrical Communication Engineering, Indian Institute of Technology, Kharagpur, in 1991 and 1993, respectively, and the Ph.D. degree from the Department of Systems Engineering, Research School of Information Sciences and Engineering, Australian National University, Canberra, in 1996.

He is currently a Professor with the Institute of Telecommunications Research, University of South Australia in Adelaide, Australia. He is also a Professor with the Dept. of Engineering Sciences in Uppsala University, Sweden, since 2013. Prior to this, he was a Professor with the Department of Electrical and Electronic Engineering, University of Melbourne, Parkville, Australia, from 2000 until early 2013. From September 1995 to September 1997, and September 1998 to February 2000, he was a Postdoctoral Research Fellow with the Department of Systems Engineering, Australian National University. From September 1997 to September 1998, he was a Postdoctoral Research Associate with the Institute for Systems Research, University of Maryland, College Park.

His current research interests include wireless communications and networks, signal processing for sensor networks, networked control systems, and molecular communication systems.

Professor Dey currently serves on the Editorial Board of IEEE Transactions on Signal Processing, IEEE Transactions on Control of Network Systems, and IEEE Transactions on Wireless Communications. He was also an Associate Editor for IEEE Transactions on Signal Processing during 2007-2010 and IEEE Transactions on Automatic Control during 2004-2007, and Associate Editor for Elsevier Systems and Control Letters during 2003-2013.

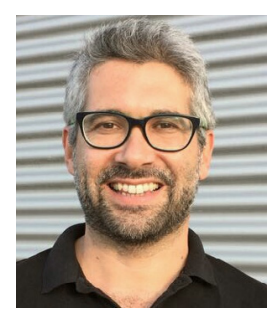

Daniel E. Quevedo is Head of the Chair of Automatic Control (Regelungs- und Automatisierungstechnik) at Paderborn University, Germany. He received Ingeniero Civil Electrónico and M.Sc. degrees from the Universidad Técnica Federico Santa María, Chile, in 2000. In 2005, he was awarded the Ph.D. degree from the University of Newcastle in Australia.

Dr. Quevedo was supported by a full scholarship from the alumni association during his time at the Universidad Técnica Federico Santa María and received several university-wide prizes upon graduating. He received the IEEE Conference on Decision and Control Best Student Paper Award in 2003 and was also a finalist in 2002. In 2009 he was awarded a five-year Research Fellowship from the Australian Research Council.

Prof. Quevedo is Associate Editor of the IEEE Control Systems Magazine, Editor of the International Journal of Robust and Nonlinear Control, and serves as Chair of the IEEE Control Systems Society Technical Committee on Networks \& Communication Systems. His research interests are in control of networked systems and of power converters. 